\title{
CONCERNING THE PRIMORDIAL ABUNDANCE OF \\ HELIUM IN QUASI-STELLAR SOURCES
}

\author{
JOSEPH SILK* \\ Princeton University Observatory, N.J., U.S.A.
}

\begin{abstract}
Primordial temperature fluctuations, which appear to be necessary in order to explain aspects of galaxy formation, can lead to observable effects in some objects. In QSS and other compact systems a correlation of absolute luminosity with primordial helium content is predicted.
\end{abstract}

It is well known that the conventional hot Friedman models of the expanding universe predict a uniform abundance for helium by number of $\sim 10$ percent relative to hydrogen. A recent study of the effect of nonuniformities in the early universe on helium production has indicated that significant reductions can be obtained in the amount of helium produced if temperature fluctuations of appreciable amplitude are assumed to be present at early epochs (Silk and Shapiro, 1970; subsequently referred to as SS). Such an assumption is necessary, in a qualitative sense, if the formation of galaxies is to be understood in the context of the Friedman cosmological models (Lifshitz, 1946). In the present contribution, I wish to remark on some rather speculative implications of this work for observations of the cosmic helium abundance in certain extragalactic systems.

In a first approximation, one may separate helium production in the hot Friedman model into two stages. At $T \gg 10^{10} \mathrm{~K}$, there is thermal equilibrium between radiation, electron-positron pairs, and neutrino pairs. The reactions

$$
n+e^{+} \leftrightarrow p+\bar{v} ; \quad p+e^{-} \leftrightarrow n+v
$$

maintain an equilibrium fraction of neutrinos, given by

$$
N_{n} / N_{p}=\exp (-Q / k T),
$$

where $Q \approx 1.3 \mathrm{MeV}$ is the energy difference between neutron and proton. At temperatures below a few times $10^{10} \mathrm{~K}$, the reactions (1) are no longer in thermodynamic balance. Peebles (1966) has shown that the neutron-proton ratio is frozen-in at $T^{*} \sim 10^{10} \mathrm{~K}$, with the residual neutron fraction given by equation (2) with $T=T^{*}$.

The second phase occurs when the neutrons capture protons, forming deuterium, at $T \sim 10^{9} \mathrm{~K}$. The subsequent reactions lead to essentially all of the remaining neutrons forming $\mathrm{He}^{4}$.

The frozen-in abundance of neutrons, and hence the primordial helium abundance, is extremely sensitive to temperature fluctuations. Table I contains the results of a computation of the effect of temperature fluctuations on a scale corresponding to the particle horizon $\sim c t$. Further details of the calculation are given in SS. Two types

* Present address: Astronomy Department, University of California, Berkeley, Calif., 94720. 
of fluctuations are considered, either random or inherently positive. Either type of fluctuation always acts in the direction of reducing the helium abundance, which has been averaged over many particle horizons. At $10^{10} \mathrm{~K}$, the scale $c t$ corresponds to a mass $\sim 10^{-2} \Omega M_{\odot}$ of matter, where $\Omega$ is the ratio of the mean matter density at the present epoch to the critical density in an Einstein-de Sitter universe. For the case of random temperature fluctuations, it is apparent that one may obtain a reduction in the primordial helium abundance by up to a factor of approximately two. The main constraint is that the excess energy density associated with the temperature fluctuations should not significantly reduce the expansion time-scale, so as to freezein the neutron abundance at a slightly lower temperature. This effect would tend to increase the primordial helium abundance.

However since the neutrons freeze in over a time-scale somewhat shorter than the expansion time at $T \sim 10^{10} \mathrm{~K}$ (Peebles, 1966), we estimate that $\tau \lesssim 1$ is the necessary condition for the consistency of our calculation.

Although primordial temperature fluctuations below a certain scale $M_{\text {crit }}$ are damped by the dissipative action of photon viscosity and diffusion at epochs prior to the decoupling of matter and radiation at $z \sim 1000$, any primordial variations in the cosmic helium abundance are maintained on all scales. This is because the main coupling between the matter and radiation is by Thomson scattering of free electrons, and Coulomb interactions effectively freeze electrons to baryons by maintaining a high degree of charge neutrality.

In order to investigate the observable consequences of primordial variations in the helium abundance, it is necessary to make further assumptions about the nature of the initial temperature fluctuations. The simplest procedure is to postulate a flat spectrum of primordial temperature fluctuations, on scales $\gtrsim 10^{-4} M_{\odot}$, and of amplitude of order unity. To avoid premature collapse of large regions of the Universe, it is necessary to assume that only subsonic vortical motions are present. As shown by Lifshitz (1946), the vortical component of velocity has constant amplitude at radiationdominated epochs of the expansion, and decays (in the absence of dissipation) as $(1+z)^{-1}$ at matter-dominated epochs. A study of the associated second-order density fluctuations leads to the result (Silk, 1971) that the power-spectrum of these irregularities generated by the turbulence peaks at a characteristic length corresponding to the particle horizon at the transition epoch between radiation - and matter dominated expansion.

One then arrives at the scheme proposed by Ozernoy and Cernin $(1968,1969)$ : At decoupling, the sound velocity decreases by many orders of magnitude, and the vortical motions become highly supersonic. Large potential motions are generated, on scales $M_{\text {crit }}<M<M_{\text {turb }}$, where $M_{\text {turb }}$ is the mass corresponding to the maximum scale over which turbulence of a given strength can generate a potential motion at the decoupling epoch. For example, when $\Omega \ll 1$, one obtains $M_{\text {crit }}=2 \times 10^{12} \Omega^{-5 / 4} M_{\odot}$ and $M_{\text {turb }}=2 \times 10^{13} \Omega^{-3 / 2} M_{\odot}$.

The potential motions generated in this manner at decoupling dissipate their excess kinetic energy by interactions with neighboring eddies. This process occurs extremely 
rapidly owing to the high efficiency of inverse Compton cooling at $z \sim 1000$ (Zeldovich and Sunyaev, 1969; cf. also Peebles, 1971). The net result is that the inevitable consequence of primordial turbulence is the formation of bound systems at $z \sim 1000$. However the primordial density fluctuations generated by the vorticity remain of small amplitude, and can lead eventually to galaxy formation at much later epochs (Silk, 1971).

It seems implausible (although not impossible) that the earliest bound systems can be proto-galaxies, since their mean density is initially $\sim 10^{-20} \Omega \mathrm{g} \mathrm{cm}^{-3}$, and considerably exceeds the mean density of $10^{-23}-10^{-26} \mathrm{~g} \mathrm{~cm}^{-3}$ observed for galactic systems (Holmberg, 1964). One could conceivably form galactic nuclei in this manner, and eject the lower density matter at subsequent epochs. Rather, we may speculate that these systems are candidates for the initial stages of quasi-stellar sources, or other compact systems. Recent work on the energy source for QSS indicates that rotation of a compact massive object may be a characteristic feature in such systems (Morrison, 1969; Cavaliere et al., 1969; Woltjer, 1970). According to these ideas, the initial rotational energy content of the system is converted to magnetic field and particle energy as the system contracts, and is accordingly an index of the bolometric luminosity of the system.

On the assumption of a flat spectrum of primordial turbulence, our work indicates that regions of primordial vortical turbulence should have a reduced primordial helium content, the reduction in helium content being correlated with the strength of the turbulence. Consequently, QSS and compact objects should show an inverse correlation, according to the present scheme, between absolute luminosity and primordial helium content.

Finally, we note that, although our assumption of random temperature fluctuations allows a reduction in the primordial helium abundance by no more than fifty percent or thereabouts, the additional hypothesis of primordial turbulence necessarily implies a net input of energy via turbulent dissipation. The resulting temperature fluctuations are inherently positive, and can therefore be considerably more effective than random temperature fluctuations in depressing the primordial helium abundance. The results of computations of the primordial helium abundance produced in the presence of

TABLE I

Fractional changes in helium abundance produced by primordial temperature fluctuations

Relative temperature fluctuation $\tau$

0.1

0.2

0.3

0.5

1.0

\begin{tabular}{ll}
$\begin{array}{l}\text { Relative change in } \\
\text { helium abundance }\end{array}$ \\
\hline Random $\tau$ & Positive $\tau$ \\
& \\
0.9 & 0.7 \\
0.8 & 0.4 \\
0.7 & 0.3 \\
0.6 & 0.1 \\
0.4 & 0.01
\end{tabular}


positive temperature fluctuations are also shown in Table I, and we see that it is possible to obtain essentially zero primordial helium in this case.

\section{Acknowledgement}

This work was supported by National Aeronautics and Space Administration grant NGL-31-001-007 to Princeton University.

\section{References}

Cavaliere, A., Pacini, F., and Setti, G.: 1969, Astrophys. Letters 4, 103.

Holmberg, E.: 1964, Ark. Astron. 3, 387.

Lifshitz, E.: 1946, J. Phys. (U.S.S.R.) 10, 116.

Morrison, P.: 1969, Astrophys. J. Letters 157, L73.

Ozernoy, L. M. and Cernin, A. D.: 1968, Soviet Astron. 11, 907.

Ozernoy, L. M. and Cernin, A. D.: 1969, Soviet Astron. 12, 901.

Peebles, P. J. E.: 1966, Astrophys. J. 146, 542.

Peebles, P. J. E.: 1971, Astrophys. Space Sci. 11, 443.

Silk, J.: 1971, Lectures presented at Institut d'Études Scientifique de Cargèse, to be published.

Silk, J. and Shapiro, S. L.: 1971, Astrophys. J. 166, 249.

Woltjer, L.: 1970, this volume, p. 277.

Zeldovich, Y. and Sunyaev, R.: 1969, Astrophys. Space Sci. 4, 301.

\section{Discussion}

Rees: The only abundance fluctuations that would survive are those on scales large compared with the horizon at the helium-formation epoch. For such fluctuations the expansion time-scale is uniquely related to the temperature, so that the concept of a 'temperature fluctuation' seems to me to make little sense.

Silk: Primordial fluctuations in the helium abundance survive on all scales larger than a characteristic scale determined by the time $t_{N}$ over which the neutrons are frozen-in. Fluctuations smaller than $L \sim c t_{N}$ undergo oscillations during the time that the neutrons are frozen-in, and consequently give rise to no net effect. This scale is approximately $L \sim c t_{N} \sim 0.1 \mathrm{ct}$ at $T \sim 10^{10} \mathrm{~K}$, and temperature fluctuations of scale $c t \gtrsim l \gtrsim L$ will produce on the average a net reduction in the number of neutrons that are frozen-in, and consequently in the abundance of $\mathrm{He}^{4}$. Since damping by photon diffusion and viscosity preserves charge neutrality, any primordial abundance fluctuations are maintained, at least until the decoupling epoch.

Petrosian: The hot spots which you mention must expand slower. How does this affect the helium formation?

Silk: The excess energy density associated with turbulent eddies acts so as to reduce the expansion time-scale. This would tend to freeze in the neutrons at a slightly lower temperature. However, provided that the relative temperature fluctuation is less than unity, the change in expansion time by this effect always remains larger than the time for neutrons to freeze-in, and the resulting reduction in the helium abundance is unaffected. 